Osteoarthritis

\title{
Chondrocalcinosis, osteophytes and osteoarthritis
}

\section{K D Brandt}

\section{Crystals, spurs, and osteoarthritis}

\section{Association between OA and chondrocalcinosis}

An association between osteoarthritis (OA) and radiographic evidence of chondrocalcinosis (CC) has been recognised for years. Crystals of calcium pyrophosphate dihydrate (CPPD) may be found in synovial fluid from patients with OA who are relatively asymptomatic as well as from those who are experiencing an acute flare up of joint pain due to an attack of pseudogout. Whether CC is the cause of OA in such people or develops as a result of changes in metabolism of the chondrocyte or in the extracellular matrix of the articular cartilage is unclear. In any event, conditions associated with CC, such as hyperparathyroidism, Wilson's disease, and haemochromatosis, are well recognised causes of "secondary" OA. $^{1}$

Chondrocytes from patients with OA who do not exhibit CC produce as much pyrophosphate as those from the cartilage of patients with CPPD disease. ${ }^{2}$ Furthermore, chondrocytes from human OA cartilage exhibit increased sensitivity to transforming growth factor $\beta$ (TGF $\beta$ ), which has been shown to induce osteophyte formation in murine knee joints, an effect not seen with insulin-like growth factor $1 .^{3}$ TGF $\beta$ also stimulates the secretion of pyrophosphate by chondrocytes, predisposing to formation of pericellular CPPD crystals; the phagocytosis of which results in the synthesis of matrix metalloproteinases by chondrocytes. ${ }^{4}$ These enzymes participate directly in the breakdown of the extracellular matrix of the cartilage and activate latent proenzymes and growth factors that cause further structural damage. Calcium crystals also decrease the synthesis of proteinase inhibitors, such as tissue inhibitors of metalloproteinases, exacerbating tissue damage. ${ }^{4}$

In addition to the effects of CPPD crystals on cartilage cited above, calcium crystals may produce direct mechanical damage to articular cartilage. Addition of CPPD crystals to the solution bathing cartilage plugs that were subjected to mechanical wear in vitro increased proteoglycan loss from the cartilage matrix, suggesting that crystals present in synovial fluid may cause increased wear of the articular surface in vivo. ${ }^{4}$ Furthermore, calcium crystals are phlogistic; their presence within the joint space and synovium may initiate an inflammatory response. $^{5}$

\section{Radiography of OA}

The paper by Neame et al in this issue of the Annals examines the association between knee OA and CC from an epidemiological perspective. ${ }^{6}$ Subjects in this cross sectional study were defined as having OA on the basis of radiographic evidence of a definite osteophyte and definite joint space narrowing (JSN, a surrogate for thinning of the articular cartilage). The authors found an association between $\mathrm{CC}$ and $\mathrm{OA}$ in the tibiofemoral and patellofemoral compartments, although patellofemoral CC was relatively uncommon and did not occur in the absence of concomitant tibiofemoral CC.

A strong association was noted between osteophytosis and JSN: a significant relationship existed between CC and both the total osteophyte score and the total number of sites within the joint affected by osteophytosis. In contrast, no association was noted between CC and JSN, leading the authors to conclude that the association between OA and CC is mediated though an association with osteophytes, rather than with JSN. However, given that calcium crystals stimulate the synthesis and release from chondrocytes of potent proteinases that can degrade the cartilage matrix (see above) and that accelerated degeneration of cartilage has been observed in joints of animals with experimentally induced OA after intra-articular injection of CPPD crystals, ${ }^{7}$ the apparent lack of association between CC and articular cartilage damage might have been due to the insensitivity of radiography for detection of CC or of the radiographic protocol employed in the epidemiological study for detection of cartilage loss (that is, JSN).

Possibly, more rigorous radioanatomical positioning than can be achieved with the conventional weightbearing extended anteroposterior view of the knee would have shown an association between CC and JSN. Considerable interest has been focused recently on the limitations of the standing anteroposterior radiograph for accurate and reproducible assessment of JSN in patients with OA. ${ }^{8}$ The issue concerns the importance of alignment of the central $x$ ray beam with the plane of the medial tibial plateau in assessment of tibiofemoral compartment joint space width, a decrease in which is generally taken as an indication of the radiographic severity of $\mathrm{OA}$ and, in serial examinations, of progression of cartilage damage. Fluoroscopically assisted positioning of the joints to align the tibiofemoral compartment (that is, with anterior and posterior margins of the medial tibial plateau superimposed $\pm 1 \mathrm{~mm}$ ), as with the technique described by Buckland-Wright, ${ }^{9}$ is associated with a more rapid rate of JSN, and smaller standard deviation of the rate of narrowing, than protocols that are less effective in providing tibial plateau alignment. ${ }^{10}$ Because of subject to subject variability in the angle of inclination of the tibial plateau relative to the horizontal plane, (fortuitous) alignment of the medial plateau with the plane of the $x$ ray beam occurs in only about $20-30 \%$ of subjects. ${ }^{11}$ Indeed, in the Nottingham study ${ }^{6}$ the reproducibility of repeated measurements of minimum joint space width on the same image $( \pm 0.31 \mathrm{~mm}$ for the left medial tibiofemoral compartment) was considerably greater than the mean annual rate of JSN in serial images of the same joint reported by several investigators (0.1-0.2 mm a year). ${ }^{10}$

What is the association between osteophytosis and OA? Although it is generally considered that radiographic evidence of definite osteophytosis is a requisite for the radiographic diagnosis of OA, ${ }^{12}$ it has been suggested that osteophytes alone-in the absence of other bony changes of OA in the radiograph (for example, subchondral sclerosis, subchondral cysts) - may merely reflect aging, and not OA. ${ }^{13} 14$ Furthermore, radiographic decreases in the interbone distance in the tibiofemoral compartment may be related to age ${ }^{15}$ raising the possibility that, had age been taken into account in the Nottingham study, a relationship between CC and joint space width might have been revealed.

\section{Chondrocalcinosis as a risk factor for OA}

Is CC a risk factor for the progression of knee OA? Can the presence of CC be used to predict which subjects with radiographic evidence of OA will progress radiographically or clinically (for example, with increasing severity of joint pain and diminution of function)? Although the presence of CPPD crystals in synovial fluid from patients with knee OA was associated with increased disability, ${ }^{16}$ and an association has been reported between the presence of CPPD crystals in 
synovial fluid and severe radiographic changes of $\mathrm{OA},{ }^{17}{ }^{18}$ others have not found an association between CC and radiographic severity of OA. ${ }^{19}$ However, these studies were all cross sectional, rather than longitudinal and, as mentioned above, the sensitivity of radiography for the detection of CPPD crystals in articular structures is poor.

In their paper, Neame et al note that increases in osteophytosis and bone remodelling were the most common changes found in patients with knee OA and CC who were followed up longitudinally. ${ }^{6}$ It is important to remember that the pathogenetic mechanisms underlying osteophytosis are different from those that result in the well recognised changes in subchondral bone in OA, in which both the formation and resorption of bone are accelerated. Furthermore, the increased turnover of subchondral bone can be inhibited by antiresorptive agents, such as bisphosphonates.

Might this be of therapeutic benefit? In an experimental canine cruciate deficiency model of $\mathrm{OA}^{20}$ in which bone turnover was markedly inhibited by administration of bisphosphonates, no effects of treatment on structural damage in the OA joint were seen. Nor did bisphosphonate treatment inhibit the prominent osteophyte formation seen in this model. However, osteophyte formation occurs by endochondral ossification-that is, it is not linked to bone resorption, in contrast with osteogenesis in subchondral bone in OA which can be modified pharmacologically-for example, by bisphosphonates. The failure of an antiresorptive drug to inhibit osteophyte formation, therefore, is not surprising. It should be noted, however, that the duration of treatment in the canine study was relatively brief. A placebo controlled clinical trial in humans is now under way whose objective is to examine whether an antiresorptive agent is effective in preventing the progression of structural damage in patients with knee OA.

\section{Diuretics and chondrocalcinosis}

Finally, the association observed by Neame et al between diuretic use and CC is interesting. ${ }^{6}$ No such association was found between CC and the use of other antihypertensive agents. Because magnesium is a cofactor for pyrophosphates that convert pyrophosphate to orthophosphate $^{21}$ and increase the solubility of CPPD crystals, ${ }^{22}$ the authors suggest that the association might have been due to iatrogenic hypomagnesaemia. It might be possible to test this hypothesis in the large prospective study of the natural history of OA soon to be initiated with support from the National Institutes of Health (NIH) and the pharmaceutical industry, in which an attempt will be made to identify surrogate biomarkers for incident $\mathrm{OA}$ and for the progression of established OA in 5000 subjects over a four year period of observation. For this purpose a cohort of subjects will be established that would be well suited to a prospective analysis examining whether diuretic treatment predisposes to CC, osteophytosis, or incident or progressive OA. Thus, the careful epidemiological observations of Neame et al relative to the relationship between $\mathrm{CC}$ and $\mathrm{OA}^{6}$ generate hypotheses that, if tested, might help answer the questions: Who gets OA and why? And, who gets clinically important OA and why?

\section{ACKNOWLEDGEMENT}

Supported in part by NIH grants AR20582, AR44370, and AR43348.

Ann Rheum Dis 2003;62:499-500

\section{Author's affiliations}

K D Brandt, Indiana University School of Medicine; Indiana University Multipurpose Arthritis and Musculoskeletal Diseases Center, 1110 West Michigan Street, Room 545, Indianapolis IN 46202, USA

Correspondence to: Professor K D Brandt; kbrandt@iupui.edu

\section{REFERENCES}

1 Brandt KD, Mankin HJ, Shulman LE. Workshop on etiopathogenesis of osteoarthritis. J Rheumatol 1986;13:112660.

2 Patrick $M$, Hamilton E, Hornby J, Doherty M. Synovial fluid pyrophosphate and nucleoside triphosphate pyrophosphatase: comparison between normal and diseased and between inflamed and non-inflamed joints. Ann Rheum Dis 1991;50:214-18.

3 Van Beuningen HM, van der Kraan PM, Arntz OJ, van den Berg WB. Transforming growth factor-B 1 stimulates articular cartilage chondrocyte proteoglycan synthesis and induces osteophyte formation in the murine knee joint. Lab Invest 1994;71:279-90.

4 Cheung $\mathbf{H}$. Calcium crystal effects on the cells of the joint: implications for the pathogenesis of disease. Curr Opin Rheumatol 2001; 12:223-7.

5 Landis R, Haskard D. Pathogenesis of crystal-induced inflammation. Curr Rheumato Rep 2001;3:36-41.
6 Neame RL, Carr AN, Muir K, Doherty M. UK community prevalence of knee chondrocalcinosis: evidence that correlation with osteoarthritis is through a shared association with osteophyte. Ann Rheum Dis 2003;62:513.

7 Fam A, Morava-Protence I, Purcell C, Young $B$, Bunring $P$, Lewis $A$. Acceleration of experimental lapine osteoarthritis by calcium pyrophosphate microcrystalline synovitis. Arthritis Rheum 1995;38:201-10.

8 Brandt KD, Mazzuca SA, Conrozier T, Dacre JE, Peterfy CG, Provvedini D, et al. Which is the best radiologic/radiographic protocol for a clinical trial of a structure-modifying drug in patients with knee osteoarthritis? Proceedings of January 17-18, 2002 Workshop in Toussus-le-Noble, France. J Rheumatol 2002;29: 1308-20.

9 Buckland-Wright JC. Protocols for radiography. In: Brandt KD, Doherty $M$, Lohmander SL, eds. Osteoarthritis. Oxford: Oxford University Press, 1998:578-80.

10 Vignon E, Piperno M, Le Graverand MP Mazzuca SA, Brandt KD, Mathieu P, et al. Measurement of radiographic joint space width in the tibiofemoral compartment of the osteoarthritic knee: comparison of standing anteroposterior and Lyon Schuss views. Arthritis Rheum 2003;48:378-84.

11 Mazzuca SA, Brandt KD, Dieppe PA, Doherty $M$, Sowers MF, Katz BP, et al. Effect of alignment of the medial tibial plateau and $x$-ray beam on apparent progression of osteoarthritis in the standing anteroposterior knee radiograph. Arthritis Rheum 2001;44:1786-94.

12 Flores RH, Hochberg MC. Definition and classification of osteoarthritis. In: Brandt KD, Doherty M, Lohmander SL, eds. Osteoarthritis. 2nd ed. Oxford: Oxford University Press. In press.

13 Danielsson L, Hernborg J. Clinical and roentgenological study of knee joints with osteophytes. Clin Orthop 1970;69:302-12.

14 Hernborg J, Nilsson BE. The relationship between osteophytes in the knee joint, osteoarthritis and aging. Acta Orthop Scand 1973;44:69-74.

15 Dacre JE, Scott DL, Da Silva JA, Welsh G Huskisson EC. Joint space in radiologically normal knees. Br J Rheumatol $1991 ; 30: 426-8$

16 Ledingham J, Regan M, Jones A, Doherty M. Radiographic patterns and associations of osteoarthritis of the knee in patients referred to hospital. Ann Rheum Dis 1993;52:520-6.

17 Dieppe P,Crocker PR, Corke CF, Doyle DV, Huskisson EC, Willoughby DA. Synovial fluid crystals. QJ Med 1979;48:533-55.

18 Schumacher H, Godon G, Paul H, Reginato A, Villanueva T, Varghese C, et al. Osteoarthritis, crystal deposition, and inflammation. Sem Arthritis Rheum 1981;12:116-19.

19 Doherty M, Watt I, Dieppe P. Pyrophosphate arthropathy - a prospective study. $\mathrm{Br} J$ Rheumatol 1984;23:141-52.

20 Myers SL, Brandt KD, Burr DB, O'Connor BL, Albrecht $M$. Effects of a bisphosphonate on bone histomorphometry and dynamics in the canine cruciate-deficiency model of osteoarthritis. J Rheumatol 1999;26:264553.

21 Rachow JW, Ryan LM. Inorganic pyrophosphate metabolism in arthritis. Rheum Dis Clin North Am 1988; 14:289-302.

22 Bennett RM, Lehr JR, McCarty DJ. Factors affecting the solubility of calcium phyrophosphate dihydrate crystals. J Clin Invest 1975;56:1571-9. 